\title{
A Novel Offline Physical Layer Impairments Aware RWA Algorithm With Dedicated Path Protection Consideration
}

\author{
Siamak Azodolmolky, Mirosław Klinkowski, Yvan Pointurier, Marianna Angelou, Davide Careglio,
} Josep Solé-Pareta, and Ioannis Tomkos

\begin{abstract}
Physical layer impairments accumulate as light propagates through a lightpath in the transparent optical networks. Therefore, it is possible to provision a lightpath, while its quality of transmission (QoT) does not meet the required threshold. Considering the physical layer impairments in the network planning phase gives rise to a set of offline Impairments Aware Routing and Wavelength Assignment (IA-RWA) algorithms. There are very few offline IA-RWA algorithms that consider dedicated path protection demands. In this work we propose a novel offline IA-RWA algorithm, called Rahyab and perform a comparative performance evaluation study, which considers two enhanced algorithms from the literature. Simulation results indicates that demand pre-processing, diverse routing, and adaptive wavelength assignment are the main reasons of lower blocking rate of Rahyab algorithm compared to the selected algorithms.
\end{abstract}

Index Terms-Dedicated path protection, impairments aware RWA, physical layer impairments, routing and wavelength assignment.

\section{INTRODUCTION}

$\mathbf{T}$ HE evolution of the optical networks as the main infrastructure underneath the emerging data-intensive applications is focused on the provisioning of more capacity in a cost-effective manner. The evolution trend depicts a transformation towards higher capacity transparent optical networks with lower (CAPEX and OPEX) cost for the next generation core networks [2]-[4].

Manuscript received October 18, 2009; revised March 21, 2010, July 05, 2010; accepted July 22, 2010. Date of publication September 02, 2010; date of current version October 06, 2010. Parts of this work have been presented in [1]. This work was supported in part by the BONE Network of Excellence ("Building the Future Optical Network in Europe") and the DICONET project ("Dynamic Impairment Constraint Optical Networking"), funded by the European Commission through the 7th ICT-Framework Program, and in part by the Polish Ministry of Science and Higher Education under the contract 643/NCOST $/ 2010 / 0$

S. Azodolmolky and M. Angelou are with the Universitat Politècnica de Catalunya (UPC), 08034 Barcelona, Catalonya, Spain, and also with Athens Information Technology (e-mail: siamak@ac.upc.edu; marianna@ac.upc.edu).

D. Careglio, and Jo. Solé-Pareta are with the Universitat Politècnica de Catalunya (UPC), 08034 Barcelona, Catalonya, Spain (e-mail: careglio@ac.upc.edu; pareta@ac.upc.edu).

Y. Pointurier and I. Tomkos are with Athens Information Technology, Peania 19002, Athens, Greece (e-mail: yvan@ieee.org, itom@ait.edu.gr).

M. Klinkowski is with National Institute of Telecommunications (NIT), 51-501 Wrocław, Poland (e-mail: m.klinkowski@itl.waw.pl).

Color versions of one or more of the figures in this paper are available online at http://ieeexplore.ieee.org.

Digital Object Identifier 10.1109/JLT.2010.2072905
To materialize the vision of transparent optical networks, while offering efficient resource utilization and strict quality of service guarantees based on certain service level agreements, the core network should efficiently provide high capacity, fast and flexible provisioning of lightpaths, high-reliability, and integrated control and management functionalities.

During the planning phase, the traffic demand is already known at least partially, enabling the network operator to perform the resource allocation task offline. Since, in all-optical networks, bandwidth is allocated under the form of lightpaths (i.e., the combination of a route between two nodes, and a wavelength), the problem of pre-planned resource allocation in such networks is called static or offline Routing and Wavelength Assignment (RWA) problem [5], [6]. The other case, whereby traffic demands are assumed to arrive in a dynamic fashion, is referred to as the online or dynamic RWA problem. This paper focuses on the offline RWA problem.

In a previous work, we performed a comprehensive literature review on the proposed algorithms that address the online and offline Impairments Aware Routing and Wavelength Assignment (IA-RWA) problem that account for physical layer impairments [7]. Indeed it is now well-known that the impact of physical layer impairments on the quality of transmission (QoT) of the lightpaths without electrical regeneration can be reduced using appropriate IA-RWA algorithms [8], and much research has been devoted to the online case. However, as indicated in [7] few works target the offline case compared with the proposed solutions for online IA-RWA problem; in addition, those proposed offline IA-RWA algorithms are evaluated for different metrics and network topologies, making them difficult to compare. Authors in [9] present a Linear Programming (LP) relaxation formulation for RWA problem that tends to yield integer solutions. The signal degradation due to physical impairments are considered as additional soft constraints on RWA. The work in [10] formulates the problem of regenerator placement and regenerator assignment in translucent optical networks, as a virtual topology design problem. Integer Linear Programming (ILP) and simple greedy heuristic algorithms are proposed to solve this problem. Once the sequence of regenerators to be used by the non-transparent connections has been determined, the initial demand set is transformed to a sequence of transparent connection requests that begin and terminate at the specified intermediate regeneration nodes. Then an IA-RWA algorithm is used to serve the converted demand set. Authors in [11] address the issue of shared protection in translucent WDM mesh networks with consid- 
eration for physical layer impairments. None of these works tackle scenarios with dedicated protected demands. A recent study shows that the CAPEX difference of shared (e.g., 1:1) and dedicated (e.g., $1+1$ ) path protection schemes is much lower in transparent optical networks comparing with its opaque counterpart [12], making dedicated protection attractive in transparent optical networks. For this reason, in this paper, we tackle the problem of offline IA-RWA where some demands can require dedicated path protection.

The contribution of this work is three-fold. First, in this paper we propose a novel IA-RWA that natively accounts for dedicated path protection; second, we enhance a selected heuristic algorithm from the literature to better include QoT related impairments and to consider the dedicated path protection [13]. Third, we enhance an ILP-based RWA formulation, from the literature [8] to include QoT requirements and also to incorporate support for protected demands and will compare their performance under similar performance evaluation framework. We show that our novel heuristic algorithm, which is called "Rahyab"", performs better than the selected algorithms under the same assumptions.

This paper is organized as follows. In Section II we briefly present the algorithms that we selected for our comparative studies. The physical layer performance evaluator as a building block of our IA-RWA framework is also explained in Section II. The enhancements to the two selected algorithms and our novel IA-RWA algorithm are discussed in Section III. The performance evaluation framework for our comparative studies and the simulation results are compiled in Section IV. Section V draws the conclusions of this work.

\section{IA-RWA FRAMEWORK}

In this section we describe selected offline IA-RWA algorithms presented in recent literature [7]. We will extend two of these algorithms in Sections III.A and III.B. Then, we present a physical layer performance evaluator, which we use as a building block for all three IA-RWA algorithms described in Section III.

\section{A. IA-RWA Algorithms}

Most of the algorithms proposed in the literature consider the online (dynamic) version of the IA-RWA problem. In contrary, there are few works in the literature regarding the offline IA-RWA problem, as we demonstrated in [7]. In general the algorithmic approach for the physical layer IA-RWA problem can be categorized either as sequential approach based on some heuristic or global optimization, which searches for an optimal solution [10]. The pros and cons of each approach are discussed in [7] and [14].

Random Search RWA (RS-RWA) is a heuristic algorithm that was proposed in [13]. The main idea in [13] is to perform a sequential search to compute lightpaths for a given random order of connection requests (permutation) in the demand set. The set of available paths is an arbitrary set of $k$ alternate shortest

\footnotetext{
${ }^{1}$ Rahyab means "path finder" in Persian language.
}

paths which are given for each pair of source destination nodes. The wavelengths are assigned according to the first-fit policy. Among a number of random order of connection requests the one that achieves the lowest lighpath blocking, is selected. Once a set of accepted lightpaths is found, the physical signal quality is verified.

ILP-RWA is an optimization-based algorithm that was studied in [8], [15]. The RWA problem is formulated as an ILP problem. It is a global optimization algorithm, which for a given set of lightpath requests finds an optimal RWA over available paths and wavelengths. The set of candidate paths consists of k-shortest paths (between each pair of nodes), which are calculated based on some impairments-aware link cost metric. The link costs correspond either to the individual impairments [8], or are calculated as a link Q factor ${ }^{2}$ [15]. The optimization criterion is the minimization of link usage subject to the network layer constraints.

An impairments-aware offline RWA algorithm that assigns Q factor costs to links before solving the problem is proposed in [16]. In that work, which is based on [8], k-shortest routes are computed considering a Q-penalty value as the link costs. Then, the wavelength that maximizes the $\mathrm{Q}$ value is selected to serve each connection request. Since the wavelength assignment is not performed jointly for all connections, a worst case assumption for the interference among lightpaths is used. Therefore, the proposed algorithm does not take into account the actual interference among lightpaths and does not truly optimize the performance, since it assumes worst case interference.

In this work we consider the RS-RWA algorithm [13] and an ILP-based RWA (ILP-RWA) algorithm [8] as two offline IA-RWA algorithms from literature [7] (both extended with path protection capability) because they use different approach to address the IA-RWA problem. Then we compare their performance with that of the new Rahyab IA-RWA algorithm under the same traffic, network and physical layer conditions.

\section{B. Physical Layer Performance Evaluator}

In the framework of transparent optical networks, physical impairments can be categorized into "static" and "dynamic" ones. Static impairments are topology-dependent, and independent of the established lightpaths in the network. In particular, in this study we account for the following static impairments: Amplifier Spontaneous Emission (ASE) noise, filter concatenation, and Polarization Mode Dispersion (PMD). Dynamic impairments depend on the presence and characteristics of other established (or to be established) lightpaths in the network. We account for the following dynamic impairments in this work: node crosstalk, originating from signal leaks at nodes, and nonlinear effects: Cross Phase Modulation (XPM) and Four Wave Mixing (FWM). To evaluate the Quality of Transmission (QoT) of a lightpath, we use a "Q-Tool", which is able to compute the "Q factor" for a lightpath given the network topology, physical characteristics, and network state (i.e., what lightpaths are already present in the network). The $\mathrm{Q}$ factor for a lightpath is

\footnotetext{
${ }^{2} \mathrm{Q}$ is a quality of transmission metric related to $\mathrm{BER}$, and will be presented in more details in Section II.B.
} 
a QoT indicator which is related to the signal's Bit-Error Rate (BER) using, for an On-Off modulated signal

$$
\mathrm{BER}=\frac{1}{2} \operatorname{erfc}\left(\frac{Q}{\sqrt{2}}\right)
$$

where the $\mathrm{Q}$ factor is defined as [17]

$$
Q=\frac{P_{1}-P_{0}}{\sigma_{1}+\sigma_{0}}
$$

where $P_{1}$ and $P_{0}$ are the means of the distributions (assumed to be Gaussian) of the received samples corresponding to the sent " 1 " and " 0 " bits, and $\sigma_{1}$ and $\sigma_{0}$ are the respective standard deviations. To estimate a $\mathrm{Q}$ factor, the $\mathrm{Q}-\mathrm{Tool}$ actually computes the following quantity:

$$
Q_{\text {est }}=\delta_{\mathrm{PMD}} \frac{P_{o p}}{\sigma_{1}+\sigma_{0}} .
$$

As suggested in [18], we model filter concatenation impairment as an eye closure penalty, yielding the term $P_{o p}$. The PMD effect is modeled as a penalty on the $\mathrm{Q}$ factor as in [19] through the multiplicative factor $\delta_{\mathrm{PMD}}$. Other impairments are accounted for through noise variances. In particular let:

$$
\begin{aligned}
& \sigma_{1}^{2}=\sigma_{1, \mathrm{ASE}}^{2}+\sigma_{1, \mathrm{XT}}^{2}+\sigma_{1, \mathrm{XPM}}^{2}+\sigma_{1, \mathrm{FWM}}^{2}, \\
& \sigma_{0}^{2}=\sigma_{0, \mathrm{ASE}}^{2}+\sigma_{0, \mathrm{XT}}^{2} .
\end{aligned}
$$

ASE noise is modeled as a noise variance according to [17] and contributes to both $\sigma_{1}$ and $\sigma_{0}$ via $\sigma_{1, \mathrm{ASE}}^{2}$ and $\sigma_{0, \mathrm{ASE}}^{2}$, respectively. The Q-Tool considers the input power of each channel for each lightpath and therefore the total input power to the amplifier in the Q-Tool considers the individual active channel's power. Since $P_{o p}, \sigma_{1, \mathrm{ASE}}$ and $\sigma_{0, \mathrm{ASE}}$ only depend on the network topology and physical parameters (as does $\delta_{\mathrm{PMD}}$ ), they can be pre-computed for fast $\mathrm{Q}$ factor estimation. We also model node crosstalk as a noise variance affecting " 1 " and " 0 " bits according to [20] via the quantities $\sigma_{1, \mathrm{XT}}^{2}$ and $\sigma_{0, \mathrm{XT}}^{2}$. The XPM effect is modeled according to [21] and accounted for within $\sigma_{1}^{2}$ via $\sigma_{1, \mathrm{XPM}}^{2}$. Similarly the FWM effect is modeled according to [22], [23] and is accounted for within $\sigma_{1}^{2}$ via $\sigma_{1, \mathrm{FWM}}^{2}$. Since node crosstalk, XPM and FWM are dynamic effects that depend on the network state, $\sigma_{1, \mathrm{XT}}^{2}, \sigma_{0, \mathrm{XT}}^{2}, \sigma_{1, \mathrm{XPM}}^{2}$ and $\sigma_{1, \mathrm{FWM}}^{2}$ have to be computed on-line by the $\mathrm{Q}$ factor estimator. For additional details about the modeling of each physical impairment, the reader is referred to [17]-[23].

\section{ENHANCEMENTS AND PROPOSED Algorithm}

As mentioned before, the main goal of this comparative study is to evaluate the performance of various offline IA-RWA algorithms, with dedicated path protection consideration. The offline IA-RWA algorithms that we presented in Section II do not consider dedicated path protection at all, therefore in Sections III.A and III.B we properly enhance two of them (respectively, [13] and [8]) to consider this additional requirement and then we compare their performances with that of a new IA-RWA algorithm, which we introduce in Section III.C. In the following, we assume that traffic demands can be either protected (i.e., the
IA-RWA algorithm must find both a primary and a backup lightpath), or unprotected.

\section{A. Enhanced RS Algorithm}

RS-RWA [13] is a heuristic IA-RWA algorithm, in which for a number of different random ordering of the connection requests (traffic demand set), the algorithm performs sequential processing of connection requests with the goal to find the lightpath assignment that achieves the lowest blocking rate. Each of these random ordering of the connection requests is considered as a permutation of the demand set. Once a set of such lightpaths is found, the physical signal quality is verified and the lightpaths that do not comply with the QoT requirements are rejected. Here we present two enhancements to improve the performance (in terms of blocking rate) of this algorithm (RS-RWA-Q) and add support for dedicated path protection (RS-RWA-QP) consideration.

The processing steps of RS-RWA are as follows:

1) Initialization: for each pair of source-destination nodes calculate $k$ alternate shortest paths.

2) Generate a permutation vector and arrange connection requests in a random order defined by the permutation vector.

3) For given permutation vector, find RWA according to the following subroutine:

a) Take first request from the permuted set of requests.

b) Select the next computed path from the set of paths.

c) Select first available wavelength on a given path according to the First-Fit policy.

d) If no wavelength is available, select next path and repeat step c); if the request is not supported by any path and wavelength, reject it.

e) Repeat steps b) to d) for all lightpath requests.

4) Repeat steps 2)-3) MaxTries times (e.g., 100) for different permutation vectors.

5) Select the RWA solution that achieves the lowest connection blocking.

6) Verify the QoT of the accepted lightpaths using a physical layer performance evaluator (Q-Tool). All lightpaths with a QoT value below a certain threshold are blocked.

Here the RWA subroutine of RS-RWA (step 3) only considers the network-layer constraints, (i.e., the availability of wavelengths on candidate paths). Moreover, the best RWA is found based on the blocking performance only at the network layer. Therefore, the performance of the physical layer is not incorporated in the RWA process and the QoT verification is performed just as a last verification step on the final RWA solution.

In order to consider the impact of physical layer impairments in this algorithm, we enhance it to RS-RWA-Q by performing a QoT verification for each permutation of demand set (i.e., after step 3 above). This modification enables us to search at step 5 for the RWA solution that achieves lowest blocking rate, among all the permutations, considering both the network layer (i.e., resource availability) and physical layer constraints.

In turn, to serve demands with dedicated path protection, we further enhance the RS-RWA-Q to obtain the RS-RWA-QP algorithm as follows. In the first step of our enhancement, in addi- 


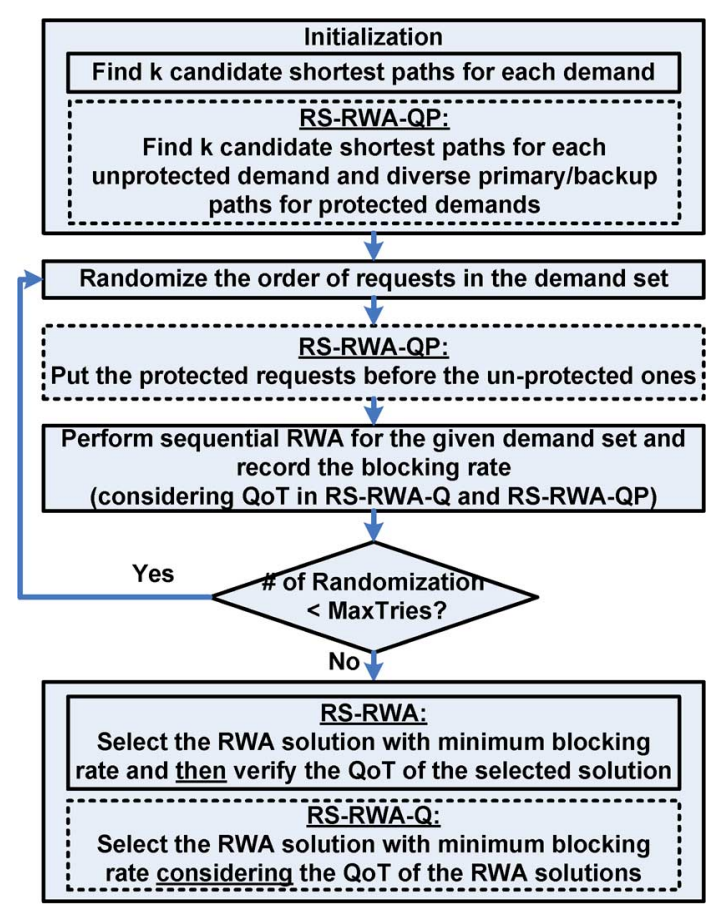

Fig. 1. Flow diagram of RS-RWA algorithm and proposed enhancements.

tion to the set of primary paths, a set of backup diverse paths is also computed (step 1). We process protected demands before unprotected demands. For each protected connection request, both primary lightpath and backup lightpath are searched similarly as in the RWA subroutine of RS-RWA. The search is performed until a pair of lightpaths is found such that one lightpath is on a primary path and the other lightpath is on the corresponding backup path. If such a pair of lightpaths cannot be established at the same time, the request is blocked. Finally, during the QoT verification phase, which is performed for each permutation, both primary and backup lightpaths are checked; if any of the two does not comply with the QoT requirements, the request is rejected. Unprotected demands are processed according to the RS-RWA-Q algorithm. The original RS-RWA and our enhancements (i.e., RS-RWA-Q and RS-RWA-QP) are indicated in the flow diagram (Fig. 1) of RS-RWA algorithm.

\section{B. ILP-Based Algorithm}

The main idea behind the ILP-RWA algorithm is to find an optimal RWA solution over a set of pre-computed paths and then perform QoT verification of candidate lightpaths using the physical layer performance evaluator (Q-Tool) presented in Section II.B. The RWA problem is formulated as a common ILP problem, i.e., subject to the network layer constraints, and physical layer constraints are not included directly into the set of constraints. Still the impairment-awareness property of the algorithm is in place with two considerations. First, the set of candidate paths is calculated based on some impairment-aware link cost metric and by means of a shortest path algorithm (e.g., the Dijkstra algorithm). The idea is to explore the paths which are less likely to experience signal distortions. Secondly, the set of lightpaths obtained after the solution of the ILP procedure is checked with respect to the optical signal quality; and if a given lightpath does not comply with the QoT requirements, it is blocked.

Here we propose extensions that decrease the blocking rate due to both the wavelength continuity and the physical layer performance constraints and also enable it to handle protected demand sets (i.e., lightpath requests with dedicated path protection). We begin with introducing the relevant notation as follows:

\section{Notations:}

$\mathcal{E} \quad$ Set of edges (directed network links).

$\mathcal{W}$ Set of wavelengths.

$\mathcal{D} \quad$ Set of demands; each demand corresponds to a pair of source-destination nodes.

$\mathcal{P}_{d} \quad$ Set of (primary) paths supporting demand $d$.

$\mathcal{P}_{d}^{\prime} \quad$ Set of backup paths supporting demand $d$.

$\mathcal{P} \quad$ Set of all paths; $\mathcal{P}=\bigcup_{d \in D}\left(\mathcal{P}_{d} \cup \mathcal{P}_{d}^{\prime}\right)$.

We assume $\left|\mathcal{P}_{d}\right|=\left|\mathcal{P}_{d}^{\prime}\right|, \forall d \in \mathcal{D}$, and for each primary path $p \in \mathcal{P}_{d}$ there is a unique backup path $p^{\prime} \in \mathcal{P}_{d}^{\prime}$ defined according to the one-to-one mapping $\gamma(p)=p^{\prime}$ such that $\gamma(q) \neq p^{\prime}, \forall q \in$ $\mathcal{P}_{d} \backslash\{p\}$. Such a mapping allows us to represent pairs of disjoint primary and backup paths.

Variables:

$x_{p w} \in\{0,1\} \quad$ A decision variable, equal to 1 if wavelength $w$ on (primary) path $p$ is assigned to an (unprotected) lightpath, and equal to 0 otherwise.

$y_{p w} \in\{0,1\} \quad$ A decision variable, equal to 1 if wavelength $w$ on primary path $p$ is assigned to a protected lightpath, and equal to 0 otherwise.

$y_{p w}^{\prime} \in\{0,1\} \quad$ A decision variable, equal to 1 if wavelength $w$ on backup path $p$ is assigned to a protected lightpath, and equal to 0 otherwise.

$x_{e w} \in\{0,1\} \quad$ An auxiliary variable, equal to 1 if wavelength $w$ on link $e$ is used, and equal to 0 otherwise.

$x_{d} \in \mathbb{Z}_{+} \quad$ A slack variable which represents the number of not-accepted unprotected lightpath requests of demand $d$.

$y_{d} \in \mathbb{Z}_{+}$ A slack variable which represents the number of not-accepted (i.e., blocked) protected lightpath requests of demand $d$.

$u \in \mathbb{Z}_{+} \quad$ A variable counting the number of links in which the most occupied wavelength (in the entire network) is used.

\section{Coefficients and Constants:}

$\delta_{e p} \quad$ A coefficient which is equal to 1 if link $e$ belongs to (primary) path $p$, and equal to 0 otherwise.

$\delta_{e p}^{\prime} \quad$ A coefficient which is equal to 1 if link $e$ belongs to

backup path $p$, and equal to 0 otherwise.

$h_{d} \quad$ The volume of (unprotected) demand $d$ (i.e., the number of lightpath requests for a given pair of nodes).

$h_{d}^{p} \quad$ The volume of protected demand $d$. 
A big constant number used as a weighting coefficient in the multi-objective function to give a priority to the blocking objective of unprotected connection requests.

$\beta \quad$ A big constant number used as a weighting coefficient in the multi-objective function to give a priority to the blocking objective of protected connection requests.

1) Basic Problem Formulation: At the beginning we present a basic ILP formulation of the RWA problem (ILP-RWA) without protected demands. The formulation has been slightly modified with respect to the one presented in [8]. The reason is that in [8] there is no link capacity constraint imposed and, as a consequence, all the requests are assumed to be served by the network. Moreover the optimization criterion is the minimization of overall link usage. Since in our study we mainly focus on blocking rate performance metric we impose additional link capacity constraints and modify the problem objective.

The ILP-RWA problem objective is to minimize the number of lightpath requests blocked due to inability to find a free wavelength

$$
\text { minimize } \quad \sum_{d \in \mathcal{D}} x_{d},
$$

subject to the following constraints.

- Input traffic constraints

$$
\sum_{p \in \mathcal{P}_{d}} \sum_{w \in \mathcal{W}} x_{p w}+x_{d}=h_{d}, \quad \forall d \in \mathcal{D} .
$$

For each demand $d$, available wavelengths on paths from set $P_{d}$ are assigned to lightpath requests $h_{d}$. Note that since $x_{p w}$ is a binary variable, each path-wavelength pair can support only one lightpath. Also the wavelength continuity constraint is imposed implicitly since decision variable $x_{p w}$ defines the entire lightpath (i.e., the assignment of wavelength $w$ on path $p$ ). Slack variable $x_{d}$ is introduced to count the number of lightpath requests that cannot be supported (i.e., blocked).

- Wavelength assignment constraints:

$$
\sum_{p \in \mathcal{P}} \delta_{e p} x_{p w}=x_{e w}, \quad \forall e \in \mathcal{E}, \forall w \in \mathcal{W} .
$$

Only one lightpath may use wavelength $w$ on link $e$ at the same time; note that since variable $x_{e w}$ is binary this bound is (implicitly) imposed.

- Range constraints

$$
\boldsymbol{x}_{p w} \in B^{|\mathcal{P}| \times|\mathcal{W}|}, \boldsymbol{x}_{e w} \in B^{|\mathcal{E}| \times|\mathcal{W}|}, \boldsymbol{x}_{d} \in \mathbb{Z}_{+}^{|\mathcal{D}|}
$$

where $B$ denotes the binary set $(B=0,1)$.

2) Formulation Enhancements: The main drawback of ILP-RWA is the lack of any (explicit or implicit) impairment-aware information involved into the optimization process. In particular any feasible RWA solution that satisfies the minimum blocking performance objective of ILP-RWA is equally good. In fact, in the presence of physical layer impairments some solutions (e.g., those that make use of a small subset of available wavelengths which, in addition, are neighbor wavelengths) may be more susceptible to crosstalk effects than the solutions that try to make use of the entire pool of wavelengths and explore wavelengths evenly. Intuitively, when the assignment of wavelengths is diversified over the network, it gives more chances for a disperse wavelength occupation in network links than, for instance, in case of first-fit assignments [24].

In order to induce the ILP algorithm to look for such solutions we reformulate the problem by introducing additional constraints on the maximal usage of a wavelength in the network and by representing the objective as a multi-objective function. This new formulation, denoted as ILP-RWA-LU, is defined as follows:

$$
\text { minimize } \quad \alpha \sum_{d \in \mathcal{D}} x_{d}+u,
$$

subject to (7)-(9), and additional maximal wavelength usage constraints

$$
\begin{aligned}
\sum_{e \in \mathcal{E}} x_{e w} & \leq u, \quad \forall w \in \mathcal{W}, \\
u & \in \mathbb{Z}_{+} .
\end{aligned}
$$

In this minimization problem we give priority to the blocking objective (we assume that $\alpha \gg 1$ ) and, in the second place, we focus on the usage of the most occupied wavelength in the network, which is represented by variable $u$ in the objective function. Constraint (11) allows to find such maximal usage, since the inequality (11) has to be satisfied for all wavelengths, and constraint (12) is integrality constraint. Note that by minimizing the maximal wavelength usage we implicitly induce the ILP solver to look for the solutions that try to balance the overall wavelength usage and, as a consequence, diversify the assignment of wavelengths in network links.

3) Protection Extensions: The ILP formulation in [8] does not take into account the existence of protected demands. Here we present an extended formulation (ILP-RWA-LUP) to handle such demands with dedicated path protection. In particular a protected connection request requires the assignment of both a primary and a backup lightpath; in case that no such simultaneous assignment is feasible, the request is blocked.

The optimization objective is formulated as

$$
\text { minimize } \quad \alpha \sum_{d \in \mathcal{D}} x_{d}+\beta \sum_{d \in \mathcal{D}} y_{d}+u \text {. }
$$

The multi-objective function of (13), which is similar the one in ILP-RWA-LU, incorporates the sum of unaccepted protected demands apart from counting the sum of unaccepted non-protected demands and the maximal wavelength usage. We consider $\beta \gg \alpha$, and $\alpha \gg 1$, so that the acceptance priority is given first to protected and then to unprotected demands.

Accordingly, constraints (7)-(9) and (11) are reformulated:

- Unprotected and protected input traffic constraints

$$
\begin{aligned}
& \sum_{p \in \mathcal{P}_{d}} \sum_{w \in \mathcal{W}} x_{p w}+x_{d}=h_{d}, \quad \forall d \in \mathcal{D} \\
& \sum_{p \in \mathcal{P}_{d}} \sum_{w \in \mathcal{W}} y_{p w}+y_{d}=h_{d}^{p}, \quad \forall d \in \mathcal{D} .
\end{aligned}
$$

Primary lightpaths are assigned to both unprotected and protected demands. 
- Wavelength assignment constraints

$\sum_{p \in \mathcal{P}} \delta_{e p}\left(x_{p w}+y_{p w}\right)$

$$
+\sum_{p \in \mathcal{P}_{d}^{\prime}} \delta_{e p}^{\prime} y_{p w}^{\prime}=x_{e w}, \forall e \in \mathcal{E}, \forall w \in \mathcal{W} .
$$

Again, only one lightpath, either primary or backup, may use wavelength $w$ on link $e$ at the same time.

- Wavelength usage constraints, which have the same application as (11) and (12) in ILP-RWA-LU formulation

$$
\begin{gathered}
\sum_{e \in \mathcal{E}} x_{e w} \leq u, \quad \forall w \in \mathcal{W}, \\
u \in \mathbb{Z}_{+} .
\end{gathered}
$$

- Backup lightpath selection constraints

$$
\begin{aligned}
& \sum_{w \in \mathcal{W}} y_{p w}-\sum_{w \in \mathcal{W}} y_{p^{\prime} w}^{\prime}=0, \\
& \forall d \in \mathcal{D}, \quad \forall p \in \mathcal{P}_{d}, p^{\prime}=\gamma(p) .
\end{aligned}
$$

Constraint (18) states that for each protected connection, a primary lightpath is assigned if and only if a backup lightpath is assigned. We remind that the selection of primary path $p$ induces the selection of backup path $p^{\prime}$ according to the mapping $\gamma(\cdot)$. Finally

$$
\begin{aligned}
& \boldsymbol{x}_{p w} \in B^{|\mathcal{P}| \times|\mathcal{W}|}, \boldsymbol{y}_{p w} \in B^{|\mathcal{P}| \times|\mathcal{W}|}, \boldsymbol{y}_{p w}^{\prime} \in B^{|\mathcal{P}| \times|\mathcal{W}|} \\
& \boldsymbol{x}_{e w} \in B^{|\mathcal{E}| \times|\mathcal{W}|}, \boldsymbol{x}_{d} \in \mathbb{Z}_{+}^{|\mathcal{D}|}, \boldsymbol{y}_{d} \in \mathbb{Z}_{+}^{|\mathcal{D}|}, u \in \mathbb{Z}_{+}
\end{aligned}
$$

which are range constraints imposed on problem variables.

\section{Rahyab IA-RWA}

In this section we present the Rahyab heuristic algorithm. We establish lightpaths in a pre-defined sequence. The order in which the demands are considered plays an important role in the performance of the proposed algorithm. Hence, the main building block in our algorithm is a demand pre-processing module. We propose two strategies to order the demands. We assess the a-priori distance $L(s, d)$ between source nodes $s$ and destination node $d$ by the length of the shortest path between $s$ and $d$. Then, we order the demands according to this shortest distance in decreasing order (demands with longest shortest path first). Ties are broken randomly in this pre-processing step. The rationale behind this is that it is generally more difficult to accommodate demands with large resource requirements, hence we seek to accommodate resource-consuming requests first, which could easily be blocked by further, less resource-consuming requests. Hence, demand pre-processing orders the static demand set in decreasing expected resource consumption, that is, protected demands have priority over unprotected ones, and within each group, we rank the demands by decreasing $L(\cdot)$.

The flow of proposed algorithm is depicted in Fig. 2. For each demand, in turn, we construct a layered network graph (LNG) as follows. The network topology for a given WDM optical network is defined by $G(V, E, W)$ where $V$ is the set of nodes in the network, $E$ is the set of bidirectional links, and $W$ is the

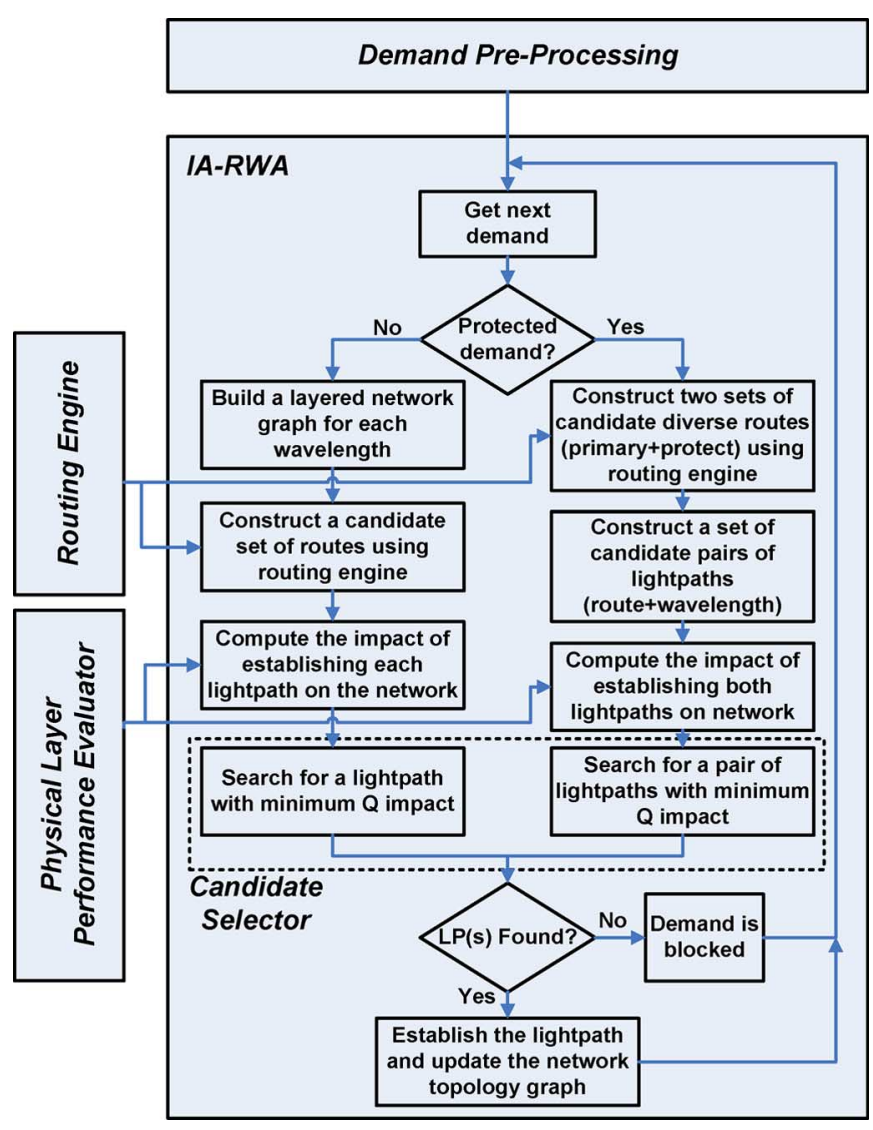

Fig. 2. Flowchart of offline Rahyab algorithm.

number of wavelengths per each fiber link. A layered network graph $\operatorname{LNG}(V, E)$ is a directed graph constructed from $G$. Each node $i \in V$ in $G$ is replicated $W$ times in the LNG. These nodes are denoted by $v_{i}(1), \ldots, v_{i}(W)$. If $L_{i, j} \in L$ connects node $i$ to node $j$, then vertices $v_{i}(w)$ and $v_{j}(w)$ are connected by two edges $e_{i, j}(w)$ and $e_{j, i}(w)$ for all $w \in W$. The representation of an LNG is shown in Fig. 3. A routing engine then constructs a set of diverse routes (maximally link- or node-disjoint, using Bhandari's algorithm [25]) in each wavelength layer of the LNG graph. This is similar to the "adaptive routing" approach [26] 3 .

After constructing the pool of candidate paths, we exploit the physical layer performance evaluator "Q-Tool" introduced in Section II.B to compute the margin of each candidate route (with respect to the minimum allowed $\mathrm{Q}$ factor $Q_{\text {threshold }}$ ) on the currently established lightpaths. The margin is computed by subtracting $Q_{\text {threshold }}$ from the $\mathrm{Q}$ factors of all active lightpaths (including the candidate path) and finding the minimum value, as expressed in (19), where $Q$ is a vector that includes the $Q$ factors of all lightpaths established in the network so far, without differentiating between primary and backup lightpaths:

$$
Q_{\text {margin }}=\min \left(\mathbf{Q}-Q_{\text {threshold }}\right) \text {. }
$$

The next step is to select a lightpath from the candidate lightpath list. We consider a heuristic, by which the candidate lightpath with highest non-negative $Q_{\text {margin }}$ is selected. If this light-

${ }^{3}$ In [26], protected demands are not handled; indeed, the notion of layered network graph is used in conjunction with shortest path routing only rather than with diverse routing. 


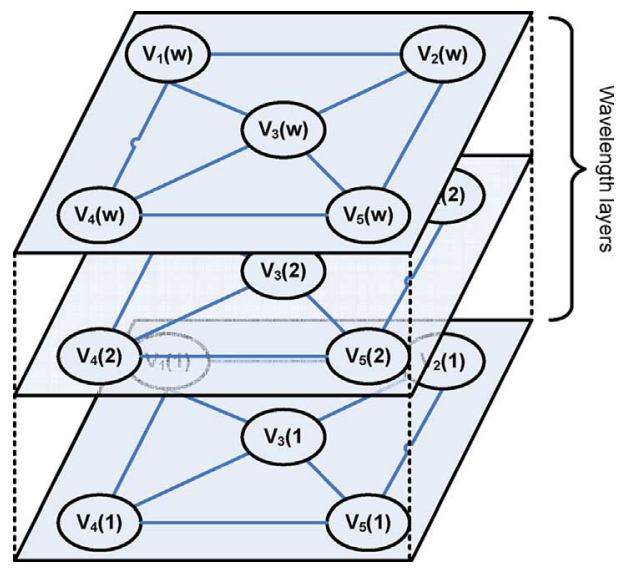

Fig. 3. Layered Network Graph.

path is found then the lightpath will be established and the network topology graph will be updated to reflect the wavelength and route allocation. Since we have decomposed the network graph into different wavelength layers and for each layer we are finding a diverse set of candidate paths, we actually find a lightpath that affects least the already active lightpaths as far as the $\mathrm{Q}$ factor metric is concerned. If a proper lightpath is not found, then the demand is blocked. For the protected demands if both primary and backup lightpaths meet the wavelength continuity and QoT constraints the demand is accepted.

\section{COMParative StUdies}

\section{A. Assumptions and Simulation Parameters}

The network topology in our simulation studies is Deutsche Telekom national network (DTNet) [27]. This network has 14 nodes and 23 bidirectional links, with an average node degree of 3.29. The line rate in this network is assumed to be $10 \mathrm{Gbps}$. We assumed a heterogeneous network topology in which the node and link architectures have different impact and contributions on physical layer impairments [27].

We define the offered load in the network as the ratio between the number of lightpath demands divided by the the number of pairs of nodes in the network $|V|(|V|-1)$. The unit traffic load corresponds to the demand set where there is a lightpath request between each pair of (distinct) source-destination nodes. However it is possible to have more than one lightpath request between a given source-destination pair. The evaluation is performed for the values of traffic load between 0.5 and 1.0 with a step of 0.1 , corresponding here to the establishment of 91 to 182 lightpaths. For each load value 50 different demand sets of random (static) lightpath requests is considered. The reported results show an average value obtained from the 50 different demand sets. The 50 demand sets are generated (at random) for each load value but the same 50 demand sets are considered as the same input sets for all algorithms. In case of protected demand sets, $20 \%$ of the demands were requesting a dedicated protection lightpath between source and destination.

The input power to the links is $-4 \mathrm{dBm}$ and $3 \mathrm{dBm}$ per channel for Dispersion Compensation Fiber (DCF) and Stan-

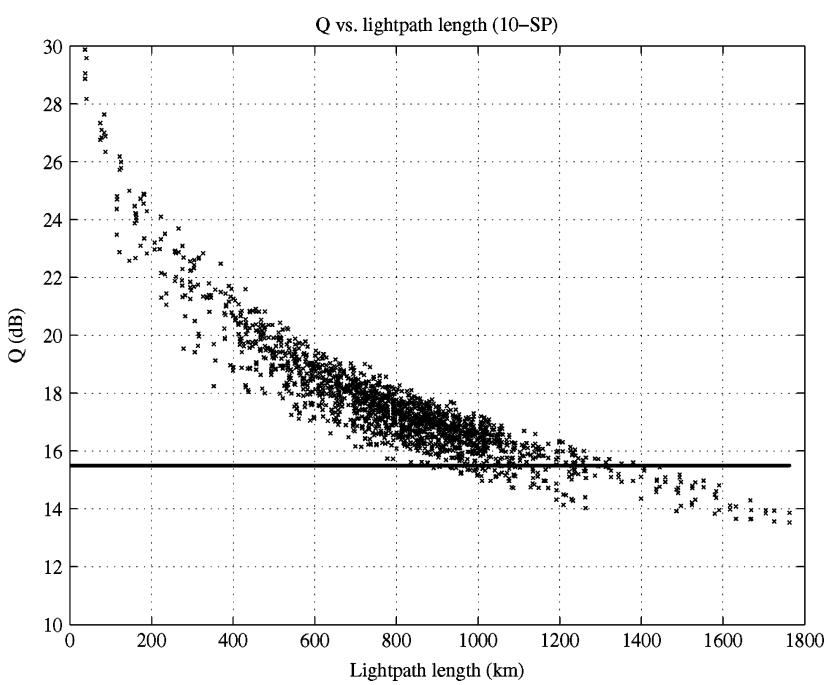

Fig. 4. Q-Factor value versus lightpath length $\left(Q_{\text {Threshold }}=15.5 \mathrm{~dB}\right)$.

dard Single Mode Fiber (SSMF) fibers respectively. We also assumed that pre-dispersion compensation of $-400 \mathrm{ps} / \mathrm{nm}$ is considered in the links. The SSMF amplifier span length in each link was set at $100 \mathrm{~km}$, followed by a DCF segment that under-compensates the dispersion of the preceding SSMF of a value of $30 \mathrm{ps} / \mathrm{nm} / \mathrm{km}$. At the end of each link the accumulated dispersion is fully compensated. It was assumed that the SSMF fibers have a dispersion parameter of $17 \mathrm{ps} / \mathrm{nm} / \mathrm{km}$ and attenuation of $0.25 \mathrm{~dB} / \mathrm{km}$. The DCF segments have a dispersion parameter of $D=80 \mathrm{ps} / \mathrm{nm} / \mathrm{km}$ and an attenuation of $0.5 \mathrm{~dB} / \mathrm{km}$. The PMD coefficient for all fiber segments is set to $0.1 \mathrm{ps} / \sqrt{\mathrm{km}}$. The channel spacing was set to $50 \mathrm{GHz}$. The noise figures that were utilized in simulation studies had a mean value of $6 \mathrm{~dB}$ with a variation of $1 \mathrm{~dB}$. In a similar manner the signal-to-crosstalk ratio had a mean value of $-32 \mathrm{~dB}$ with a deviation of $2 \mathrm{~dB}$ around this mean value in each node. The threshold value for computing the impact on $\mathrm{Q}$ factor (i.e., $Q_{\text {threshold }}$ ) is $15.5 \mathrm{~dB}$ (corresponding to BER $=10^{-9}$ without FEC). In Fig. 4 the $\mathrm{Q}$ factor value of 10 shortest paths between all possible pairs of the nodes in the network is depicted. Without considering the impact of other established lightpaths, the maximum optical reach is about $1500 \mathrm{~km}$ in this network.

We implemented two versions of the RS-RWA algorithm [13] as detailed in Section II (plain algorithm) and the enhanced versions as detailed in Section III.A. In both cases MaxTries(= 100) permutations of each demand set are examined. The candidate shortest paths (SP) are calculated considering the link length as the link cost metric. The number of paths that we consider between each pair of source-destination nodes is $k=$ $\{2,10\}$ for RS-RWA, $k=10$ for RS-RWA-Q, and $k=\{1,2\}$ for ILP-RWA (see Sections II and III.B) and $k=\{1,2,3\}$ in ILP-RWA-LU. Note that for $k=1$ the routing sub-problem of ILP-RWA is relaxed and the algorithm performs as a wavelength assignment algorithm. We assume $\alpha=|\mathcal{E}|+1$ and $\beta=$ $\alpha\left(\sum_{d \in \mathcal{D}} h_{d}+1\right)+1$. The Rahyab algorithm (see Section III.C) considers $k=10$ shortest path between each source-destination pair in its candidate set and if the demand is protected, $k=2$ diverse routes between each pair are computed. 


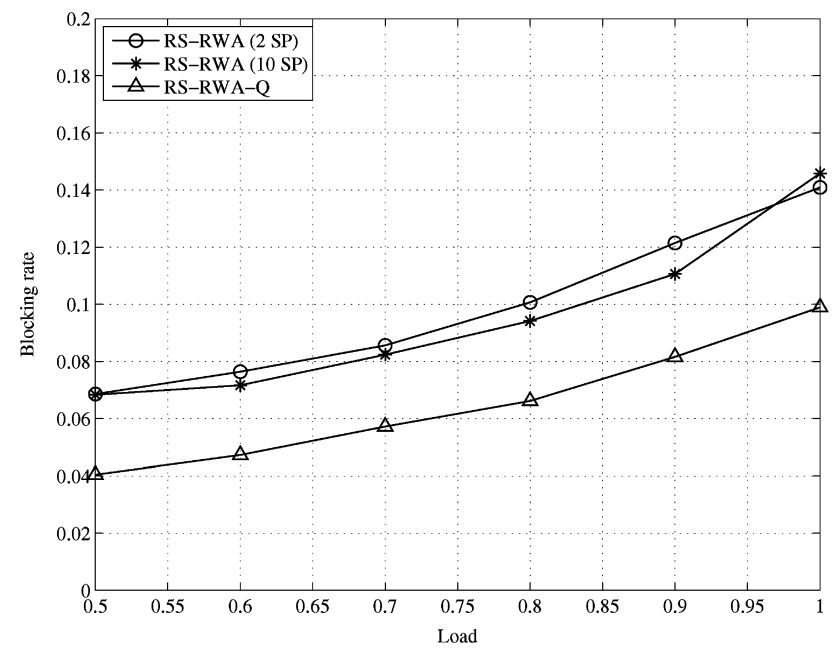

Fig. 5. Blocking rate versus various load value for three variations of RS-RWA algorithm (number of channels per link $W=16$ ).

\section{B. Results}

In order to compare the performance of the selected algorithms the blocking rate of demand sets is considered as our key performance metric. Blocking rate is the ratio of number of blocked demands to the total number of demands in a given demand set. More specifically, metrics that characterize the quality of the solutions obtained by the IA-RWA algorithms are: a) Blocking rate for a given number of wavelengths as a function of the traffic load and b) Blocking rate for a given load as a function of the number of channels per link.

The blocking rates of three variations of RS-RWA algorithm are shown in Fig. 5. The performance of the enhanced RS-RWA is clearly better than the original RS-RWA algorithm (with $k=2$ and $k=10$ shortest path computation). For instance at Load $=0.7$ the performance of RS-RWA-Q algorithm is $44 \%$ better than RS-RWA (10 SP). The main reason for this improvement is the consideration of physical layer impairments for each permutation of the demand set. Therefore, the RS-RWA-Q selects the RWA solution that achieves the lowest blocking rate among the other candidate permutations, while in RS-RWA algorithm, the RWA decisions are made without consideration for the performance of the physical layer.

This improvement is also observed when we considered the blocking rate of RS-RWA algorithms as a function of available channels per fiber link for a specific load value in the network. The result of this experiment is depicted in Fig. 6. Even with more channels per link, the blocking rates of the RS-RWA-Q algorithms do not decrease. The small fluctuation in blocking rate is due to the slightly different contribution of crosstalk that could occur for each scenario of available channels per fiber. This behavior is mainly caused by the first-fit wavelength assignment policy in RS-RWA-Q, which always try to allocate successive (neighbor) wavelengths, no matter how many wavelengths are available in the link, thus results in the crosstalk effect and eventually same level of blocking rate.

We performed the same study among the various ILP-RWA algorithms. Fig. 7 depicts the blocking rate of different variations of ILP-RWA and ILP-RWA-LU algorithms. We can ob-

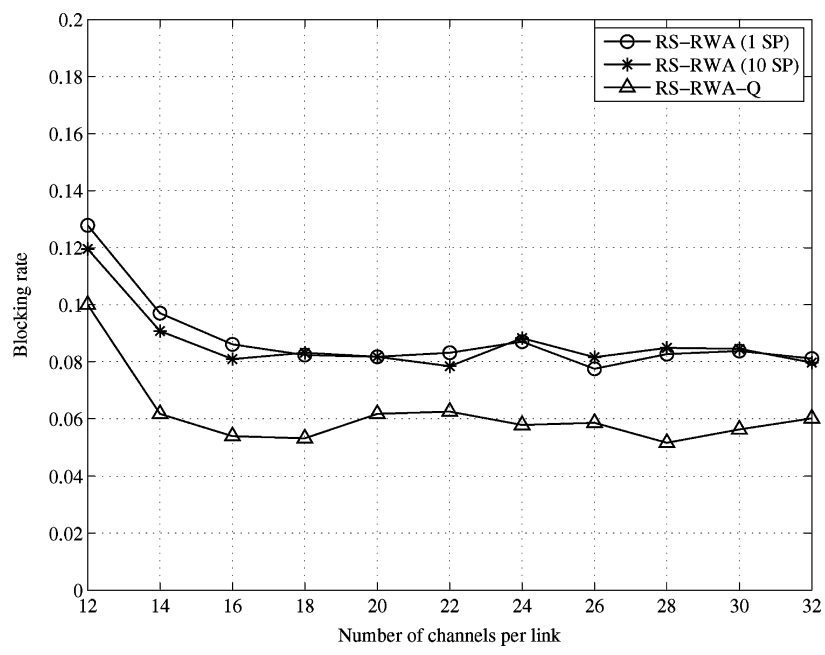

Fig. 6. Blocking rate versus number of channels per fiber link $(\operatorname{Load}=70 \%)$.

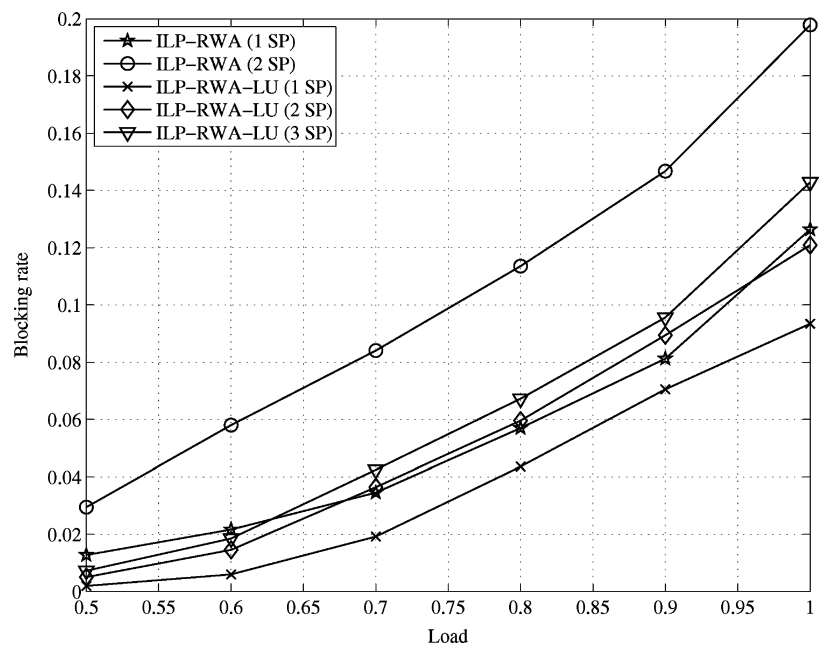

Fig. 7. Blocking rate versus Load for five variations of ILP-RWA algorithms $(W=16)$

serve that the performance of the ILP-RWA-LU (1 SP) is better than the original ILP-RWA algorithms (both 1 SP and 2 SP). Another important observation in this result indicates that increasing the number of candidate shortest paths also increases the blocking rate of ILP-RWA and ILP-RWA-LU algorithms. Increasing the size of the candidate paths helps the ILP-RWA algorithm to easier find a globally optimized RWA solution, for which the satisfaction of the QoT requirement is not guaranteed. Larger pool of candidate paths, coupled with lack of QoT verification paves the way for higher probability of picking a candidate path that will not satisfy the QoT requirement. Better performance can be observed for the ILP-RWA-LU algorithm, in which an additional objective (the usage of the same wavelength in the network), is introduced. This objective makes the algorithm diversify the assignment of wavelengths and therefore decrease the impact of physical impairments that are caused due to the lightpaths crosstalk.

In Fig. 8 the blocking rate performance of various ILP-RWA algorithms for a given amount of load in the network is depicted as a function of the available channels per fiber link. In general by increasing the number of available resources per each fiber 


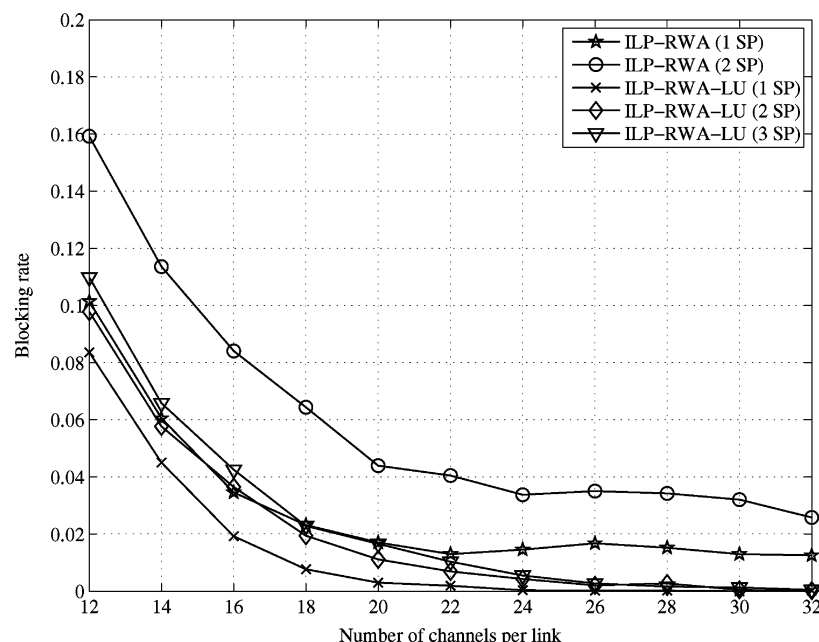

Fig. 8. Blocking rate versus number of channels per link for five variations of ILP-RWA algorithms ( Load $=70 \%)$.

link the chance of accommodating the given load in the network is increased, this can be therefore translated to lower blocking rate. The ILP-RWA-LU assigns wavelengths more diversely that causes lower interaction between neighboring lightpaths and thus lower blocking rate. Again note that increasing the number of candidate paths does not improve the performance of different variations of the ILP-RWA-LU algorithms. As mentioned before, increasing the number of candidate paths simply helps the ILP formulation to find an RWA solution, which does not necessarily satisfy the QoT requirement.

After identifying the impact of our enhancements in RS-RWA and ILP-RWA algorithms, we repeated our performance evaluation study considering the Rahyab algorithm. In Figs. 9 and 10 the blocking rate of selected IA-RWA algorithms is presented. In spite of our enhancements, the RS-RWA-Q algorithm has the worst performance compared with other algorithms. The main reasons for this low performance reside in the random selection of the demand set, without considering a particular order for demand processing and also the first-fit wavelength assignment policy utilized in this algorithm. The first-fit policy simply ignores the negative impact of assigning neighboring channels on lightpath (with potentially many common links). The ILP-RWA algorithm finds a globally optimized RWA solution. This global optimization policy performs well for low load value, however by increasing the load, the probability of finding a globally optimized solution that does not satisfy the QoT requirement increases too. This is observed for the loads 0.9 and 1.0, in which the performance of the ILP-RWA is comparable or even worse than the RS-RWA algorithm. The ILP-RWA-LU enhancement introduces an additional objective (the maximal usage of a wavelength), which makes the algorithm diversify the assignment of wavelengths and leads to lower blocking rate. In case of unprotected demands only, obtained results show that the blocking after the ILP procedure is lower if more SPs are available (e.g., we have 0 blocking with 3 SPs versus 5\% blocking with 1 SP, under $100 \%$ load). On the other hand, quality blocking is much higher for scenarios with more SPs given (adequately, 14\% for 3 SP versus $4 \%$ for $1 \mathrm{SP})$. Then, the overall blocking, which is the sum of both

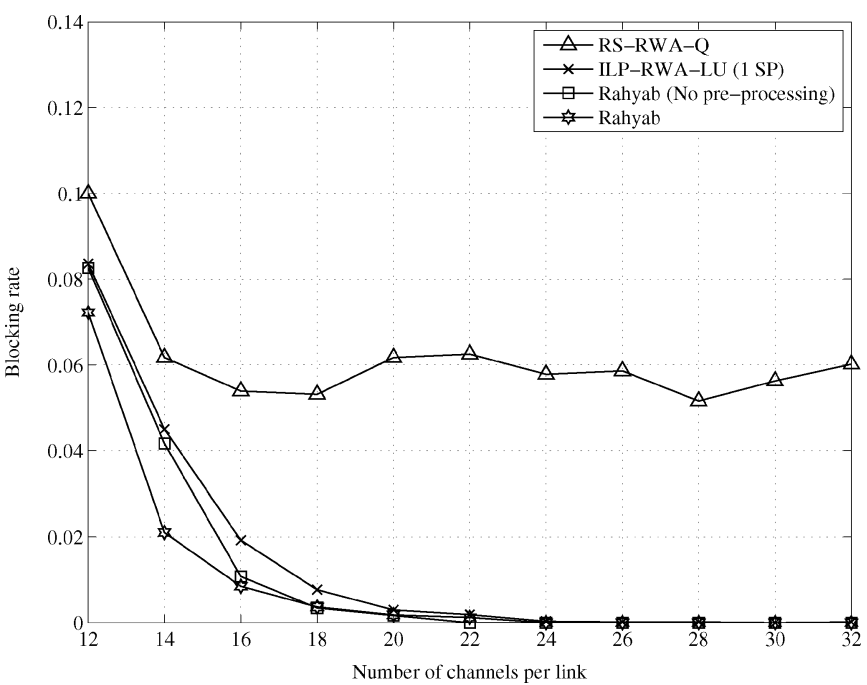

Fig. 9. Blocking rate versus load for selected IA-RWA algorithms $(W=16)$.

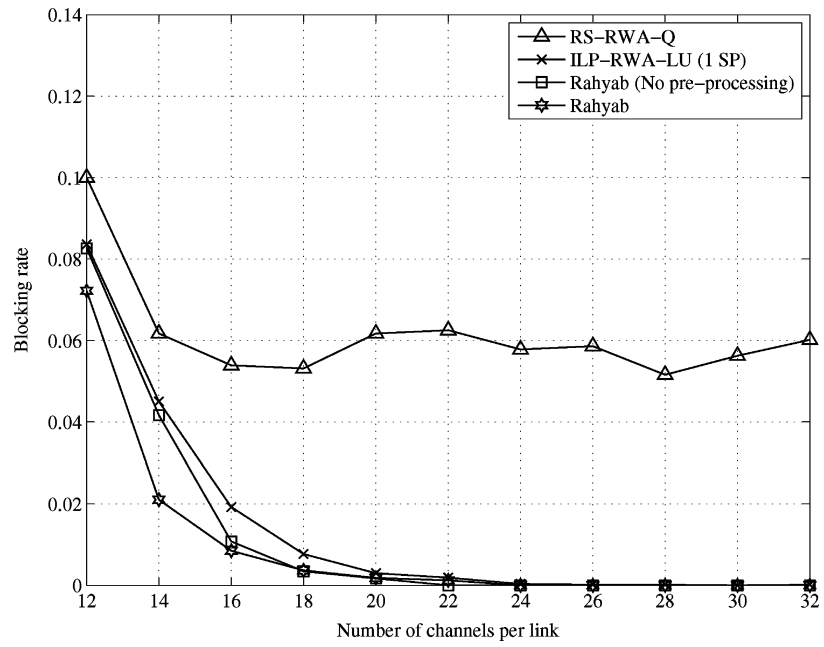

Fig. 10. Blocking rate versus number of channels per link for selected IA-RWA algorithms $(\operatorname{Load}=70 \%)$.

blocking components, leads to the presented results. Finally the Rahyab algorithm performs better than selected algorithms due to several reasons. The demand pre-processing part of Rahyab rearranges the order of the demands in the demand set in a way that demands that require more resources (i.e., longest shortest path first) are processed first. The impact of the demand pre-presseing can be observed in these figures for two cases of Rahyab algorithm (with and without demand pre-processing). The wavelength assignment policy in Rahyab considers the impact of establishing the new lightpath on all the already established lightpaths. This adaptive wavelength assignment, with the goal of establishing a lightpath with minimum impact on other established lightpaths gives more room to accommodate lightpaths in the network, and leads to lower blocking rate. The k-shortest path engine enables Rahyab to find a rich set of candidate lightpaths between source and destination.

The downside of Rahyab is the extensive utilization of the Q-Tool, which itself is very computationally intensive; however, since the working setup is for offline network dimensioning, 


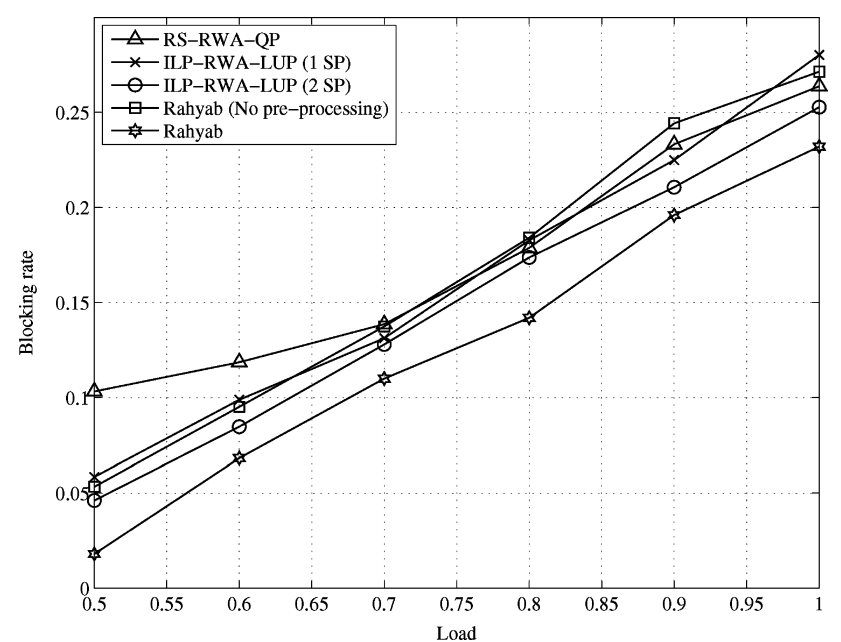

Fig. 11. Blocking rate versus load for selected IA-RWA algorithms $(W=16)$ and $20 \%$ protected demands.

computation time is of secondary importance and Rahyab is adapted to offline network design.

The next set of our results summarizes the blocking rate performance of selected algorithms with consideration of protected demands. In Figs. 11 and 12 the blocking rate of the selected algorithms versus load and also versus number of channels per fiber link is depicted. In order to reveal the impact of pre-processing phase of Rahyab algorithm, we have also included the results of Rahyab algorithm, without demand pre-processing step. For small to medium amount of load, the diverse routing engine and adaptive wavelength assignment module compensate for the lack of pre-processing block. However by increasing the load, the mentioned components of the Rahyab algorithm are not able anymore to avoid higher blocking rate. We observe in Fig. 12 the impact of Rahyab pre-processing module when in particular there are low number of channels per fiber. However by increasing the number of channels per fiber, the diverse routing engine and adaptive wavelength assignment compensate for the lack of pre-processing module. We observed the same impact in other comparisons but it is only reported in these two figures to maintain the completeness of results and clarity.

The blocking rate of ILP-RWA-LUP (2 SP) is higher than Rahyab algorithm but lower than RS-RWA-QP. In case of ILP-RWA-LUP, our simulation results revealed that the wavelength availability-dependent blocking is a significant blocking factor in the dedicated path protection scenario. ILPRWA-LUP (2 SP) performs slightly better than ILPRWA-LUP (1 SP) since it has a higher chance to find the primary and backup path when performing the ILP procedure. The results for ILP-RWA-LUP (2 SP) and ILP-RWA-LUP (3 SP) (not reported here) are very similar and only slightly vary for different load values. Fig. 12 shows that the Rahyab algorithm is able to serve all demands when the number of channels per link is $W=28$. RS-RWA-QP algorithm utilizes a first-fit wavelength assignment policy, which tries to allocate wavelengths with a predefined order. This increases the impact of crosstalk related impairments and therefore the blocking rate does not reach the non-blocking $(0 \%)$ level.

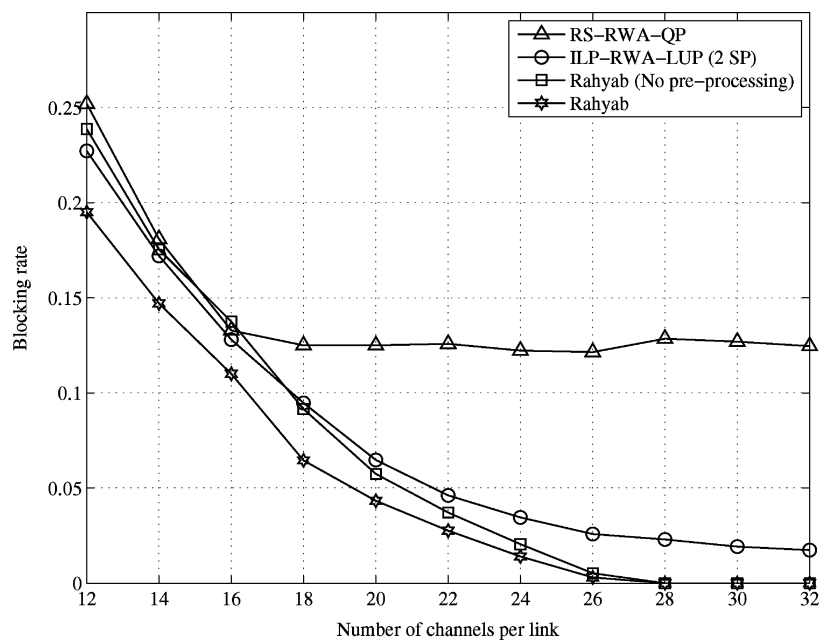

Fig. 12. Blocking rate versus number of channels per link for selected IA-RWA algorithms ( Load $=70 \%)$ and $20 \%$ protected demands.

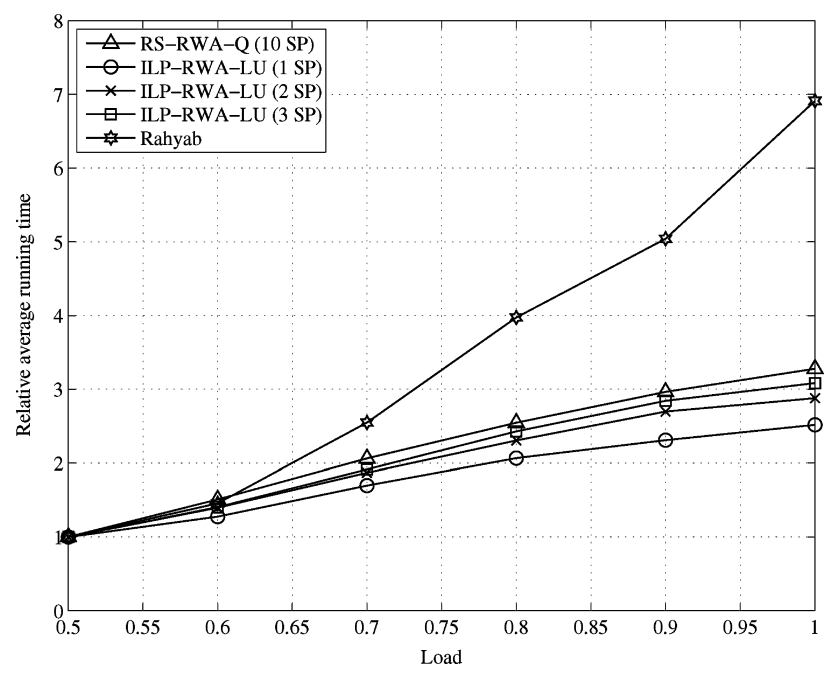

Fig. 13. Relative average running time versus load $(W=16)$.

In order to evaluate the time complexity and scalability of the algorithms we defined the relative average running time performance metric. This metric is the ratio of the average running time of a given algorithm for a certain load $\rho$ over the average running time of the same algorithm for the reference load (i.e., $\rho=0.5$ ). This relative metric removes the dependency of the running time of an algorithm to the performance of a particular hardware platform. The relative average running time of ILP-RWA-LU, RS-RWA-Q, and Rahyab are depicted in Fig. 13. The ILP-RWA-LU has the best relative running time compared to RS-RWA-Q and Rahyab algorithms. The absolute running time of RS-RWA-Q, and three variations of ILP-RWA-LU algorithms (i.e., 1 SP, 2 SP, and 3 SP) was 138, 27, 32 and 38 seconds respectively for the reference load. The running time of Rahyab for the same load value (under virtual machine setup/execution) was approximately 14 hours.

The ILP-RWA-LU belongs to the global optimization techniques, in which the impact of the physical layer impairments is indirectly considered in the ILP formulation. Its relative running time depends on the performance of the utilized ILP solver 
and the performance of the Q-Tool for verification step. The complexity of the RS-RWA-Q algorithm is dominated by the required time for verification of QoT of the candidates. However as the load increases the size of the demand set is increased and the final QoT evaluation is more time consuming. Rahyab algorithm intensively utilizes the Q-Tool and given the complexity of the analytical models inside the Q-Tool the running time of Rahyab is much higher than other selected algorithms. For a single unprotected demand, the maximum number of Q-Tool invocation is equal to the number of candidate paths in each $\mathrm{LNG}$ (i.e., $k \times W$ in which $k$ is the number of candidate paths and $W$ is the number of wavelengths per link). The dominant impairment in term of computation complexity in Q-Tool is the FWM module, in which the combinatorial impact of three possible channels on a given channel is explored. The computation time of Q-Tool is increased in the polynomial order by increasing both the number of active lightpaths that are passed to the Q-Tool and by increasing the number of available channels per fiber.

\section{CONCLUSION}

The offline IA-RWA algorithms play an important role in serving the demands with possibly minimum amount of blocking. In this work we evaluated the RS-RWA heuristic and our enhancements (RS-RWA-Q, RS-RWA-QP), the ILP-RWA and our enhancements (ILP-RWA-LU, ILP-RWA-LUP), along with a novel algorithm (called Rahyab). Our enhancement to the RS-RWA heuristic (i.e., RS-RWA-Q) reduced the blocking rate of demands by an average of $35 \%$ for different loads compared to the original scheme (i.e., RS-RWA). The performance of ILP-RWA-LU (1 sp) formulation (i.e., enhanced ILP-RWA) is also improved by $71 \%$. The Rahyab algorithm performs better than the other two algorithms with respect to the blocking rate performance metric. For instance when the offered load to the network is $80 \%$, the blocking rate of Rahyab algorithm is decreased by $61 \%$ and $42 \%$ compared to RS-RWA-Q and ILP-RWA-LU algorithms respectively. When the number of channels were limited only to 14 channels per link (i.e., $W=14$ ) and the demand set included both the protected and un-protected demands, the performance of Rahyab algorithm was better than RS-RWA-QP and ILP-RWA-LUP by $22 \%$ and $15 \%$ respectively. RS-RWA-Q and RS-RWA-QP algorithms find an optimum permutation of demand set that leads to lower blocking rate, however proper ordering of demands are not considered in it. Furthermore the wavelength assignment policy in this algorithm is first fit, which increases the chance of unwanted crosstalk between neighboring lightpaths. ILP-RWA-LU performs better than RS-RWA-Q, mainly thanks to the diversification of wavelength assignments, however the adaptive wavelength assignment and proper ordering of the demand set in Rahyab helps it perform better compared to its ILP-based counterpart.

The demand pre-processing technique can be further enhanced with more sophisticated schemes. One possible approach could be the use of global optimization (e.g., liner programming) to find an optimum order for demands. Proper integration of IA-RWA algorithms with control plane and its impact on control plane operation are among our ongoing research activities.

\section{ACKNOWLEDGMENT}

Authors would like to thank Dr. Matthias Gunkel for providing the detailed DT network topology and the reviewers for their constructive comments.

\section{REFERENCES}

[1] S. Azodolmolky, Y. Pointurier, M. Angelou, J. Solé-Pareta, and I. Tomkos, "An offline impairment aware RWA algorithm with dedicated path protection consideration," in Proc. IEEE/OSA OFC, Mar. 2009, Paper OWI1.

[2] J. Berthold, A. A. M. Saleh, L. Blair, and J. M. Simmons, "Optical networking: Past, present, and future," J. Lightw. Technol., vol. 26, no. 9, pp. 1104-1118, May 2008.

[3] S. Sygletos, I. Tomkos, and J. Leuthold, "Technological challenges on the road toward transparent networking," J. Opt. Netw., vol. 7, no. 4, pp. 321-350, Apr. 2008.

[4] S. Azodolmolky et al., "A dynamic impairment aware networking solution for transparent mesh optical networks," IEEE Commun. Mag., vol. 47, no. 5, pp. 38-47, May 2009.

[5] R. Ramaswami and K. N. Sivarajan, "Routing and wavelength assignment in all-optical networks," IEEE/ACM Trans. Netw., vol. 5, no. 3, pp. 489-500, Oct. 1995.

[6] H. Zang, J. P. Jue, and B. Mukherjee, "A review of routing and wavelength assignment approaches for wavelength-routed optical \{WDM networks," Opt. Netw. Mag., vol. 1, pp. 47-60, Jan. 2000.

[7] S. Azodolmolky, M. Klinkowski, E. Marin, D. Careglio, J. Solé-Pareta, and I. Tomkos, "A survey on physical layer impairments aware routing and wavelength assignment algorithms in optical networks," Elsevier Comput. Netw., vol. 53, no. 7, pp. 926-944, May 2009.

[8] P. Kulkarni, A. Tzanakaki, C. Mas Machuka, and I. Tomkos, "Benefits of $Q$-factor based routing in WDM metro networks," in Proc. ECOC, Sep. 2005, vol. 4, pp. 981-982.

[9] K. Manousakis, K. Christodoulopoulos, and E. Varvarigos, "Impairment-aware offline rwa for transparent optical networks," Proc. INFOCOM, pp. 1557-1565, Apr. 2009.

[10] K. Manousakis, K. Christodoulopoulos, E. Kamitsas, I. Tomkos, and E. A. Varvarigos, "Offline impairment-aware routing and wavelength assignment algorithms in translucent WDM optical networks," J. Lightw. Technol., vol. 27, no. 12, pp. 1866-1877, Jun. 2009.

[11] X. Yang, L. Shen, and B. Ramamurthy, "Survivable lightpath provisioning in WDM mesh networks under shared path protection and signal quality constraints," J. Lightw. Technol., vol. 23, no. 4, pp. 1556-1567, Apr. 2005

[12] D. Staessens, D. Colle, M. Pickavet, and P. Demeester, "Path protection in WSXC switched networks," in Proc. ECOC, Sep. 2008, pp. 1-2.

[13] M. Ali Ezzahdi, S. Al Zahr, M. Koubaa, N. Puech, and M. Gagnaire, "LERP: A quality of transmission dependent heuristic for routing and wavelength assignment in hybrid WDM networks," in Proc. ICCCN, Oct. 2006, pp. 125-136.

[14] J. M. Simmons, Optical Network Design and Planning. New York: Springer, 2008.

[15] I. Tomkos, D. Vogiatzis, C. Mas, I. Zacharopoulos, A. Tzanakaki, and E. Varvarigos, "Performance engineering of metropolitan area optical networks through impairment constraint routing," IEEE Commun. Mag., vol. 42, no. 8, pp. S40-S47, Aug. 2004.

[16] G. Markidis, S. Sygletos, A. Tzanakaki, and I. Tomkos, "Impairment aware based routing and wavelength assignment in transparent long haul networks," in Proc. IFIP ONDM, May 2007, pp. 48-57.

[17] G. P. Agrawal, Fiber-Optic Communications Systems, 3rd ed. New York: Wiley, 2002.

[18] S. Norimatsu and M. Maruoka, "Accurate $Q$-factor estimation of optically amplified systems in the presence of waveform distortion," $J$. Lightw. Technol., vol. 20, no. 1, pp. 19-27, Jan. 2002.

[19] C. D. Cantrell, "Transparent optical metropolitan-area networks," in Proc. IEEE LEOS, Oct. 2003, vol. 2, pp. 608-609.

[20] B. Ramamurthy, D. Datta, H. Feng, J. P. Heritage, and B. Mukherjee, "Impact of transmission impairments on the teletraffic performance of wavelength-routed optical networks," J. Lightw. Technol., vol. 17, no. 10, pp. 1713-1723, Oct. 1999. 
[21] V. T. Cartaxo, "Cross-phase modulation in intensity modulation-direct detection WDM systems with multiple optical amplifiers and dispersion compensators," J. Lightw. Technol., vol. 17, no. 2, pp. 178-190, Feb. 1999.

[22] W. Zeiler, F. Di Pasquale, P. Bayvel, and J. E. Midwinter, "Modelling of four-wave mixing and gain peaking in amplified WDM optical communication systems and networks," J. Lightw. Technol., vol. 14, no. 9, pp. 1933-1942, Sep. 1996.

[23] K. Inoue, K. Nakanishi, and K. Oda, "Crosstalk and power penalty due to fiber four-wave mixing in multichannels transmissions," J. Lightw. Technol., vol. 12, no. 8, pp. 1423-1439, Aug. 1996.

[24] J. He, M. Brandt-Pearce, Y. Pointurier, and S. Subramaniam, "Adaptive wavelength assignment using wavelength spectrum separation for distributed optical networks," in Proc. ICC, Jun. 2007, pp. 2406-2411.

[25] R. Bhandari, Survivable Networks: Algorithms for Diverse Routing, 1st ed. Norwell, MA: Kluwer, 1999.

[26] A. Mokhtar and M. Azizoĝlu, "Adaptive wavelength routing in all-optical networks," IEEE/ACM Trans. Netw., vol. 6, no. 2, pp. 197-206, Apr. 1998.

[27] D. Staessens et al., "Definition of dynamic optical network architectures," DICONET Deliverable D2.1 Dec. 2008 [Online]. Available: http://www.diconet.eu/deliverables.asp

Siamak Azodolmolky received the B.Eng. degree from Tehran University, Tehran, Iran, in 1994 and the M.Eng. in computer architecture from Azad University, in 1998.

He has worked with the Data Processing Iran during 1992-2001. He received his second M.Sc. degree from Carnegie Mellon University, in 2006. He joined Athens Information Technology as a researcher in 2007, while also pursuing a $\mathrm{Ph} . \mathrm{D}$. He is a professional member of ACM and a student member of IEEE.

Mirosław Klinkowski received the M.Sc. degree from Warsaw University of Technology, Poland, in 1999 and the Ph.D. degree from Universitat Politècnica de Catalunya (UPC), Barcelona, Spain, in 2008.

$\mathrm{He}$ is an Assistant Professor in the Department of Transmission and Optical Technologies at the National Institute of Telecommunications in Warsaw, Poland, and is a Collaborating Researcher at the Universitat Politècnica de Catalunya (UPC), Barcelona, Spain. His publications include several book chapters and more than 50 papers in relevant research journals and refereed international conferences. He has participated in many European projects dealing with topics in the area of Optical Networking. He is currently involved in COST 2100 and COST IC0804 actions. His research interests include optical and wireless networking with emphasis on network modeling, design, and performance analysis.

Yvan Pointurier (S'02-M'06) received the Diplôme d'Ingénieur from Ecole Centrale de Lille, France in 2002, the M.S. degree in computer science in 2002, and a the Ph.D. degree in electrical engineering in 2006, both from the University of Virginia.

He spent two years at McGill University in Montreal, Canada as a Postdoctoral Fellow and then one year at Athens Information Technology, Greece, as a technical leader for a European Project. In 2009 he joined Alcatel-Lucent Bell Labs, France, as a Research Engineer. His research interests span design, optimization and monitoring of networks in general, and optical networks in particular.

Dr. Pointurier is a co-recipient of the Best Paper Award at the IEEE ICC 2006 Symposium on Optical Systems and Networks.
Marianna Angelou received the undergraduate degree in computer science from the Aristotle University of Thessaloniki, Greece, in 2005, and the M.Sc. degree in information and telecommunication technologies from Athens Information Technology in 2008. She is currently a Ph.D. candidate in the Computer Architecture department of the Polytechnic University of Catalonia.

Her research activities focus on cross-layer optimization techniques for optical networks.

Davide Careglio (S'05-M'06) received the M.Sc. and Ph.D. degrees in telecommunications engineering from the Universitat Politècnica de Catalunya (UPC), Barcelona, Spain, in 2000 and 2005, respectively, and the Dr.Ing. degree in electrical engineering from Politecnico di Torino, Turin, Italy, in 2001.

$\mathrm{He}$ is an Associate Professor in the Department of Computer Architecture at UPC. Since 2000, he is a staff member of the CCABA and of the Broadband Communications Research (CBA) group. He has participated in several national and EU funded research projects and cost actions including four running FP7 projects. Together with his colleagues and students he has authored more than 100 peer-reviewed articles. His research interests are in the fields of optical networks with emphasis on packet-based switching technologies, quality of service $(\mathrm{QoS})$ provisioning, energy-efficiency, and multi-layer traffic engineering. He has participated in the technical program committees of several conferences, including IEEE ICC and IEEE Globecom.

Josep Solé-Pareta received the M.Sc. degree in telecom engineering in 1984, and the Ph.D. degree in computer science in 1991, both from the Technical University of Catalonia (UPC), Barcelona, Spain.

In 1984 he joined the Computer Architecture Department of UPC. Currently he is Full Professor with this department. He did a Postdoc stage (summers of 1993 and 1994) at the Georgia Institute of Technology. His publications include several book chapters and more than 100 papers in relevant research journals $(>$ 25 ), and refereed international conferences. His current research interests are in Nanonetworking Communications, Traffic Monitoring, Analysis and High Speed and Optical Networking and Energy Efficient Transport Networks.

Ioannis Tomkos received the B.Sc. degree from the University of Patras, Greece, and the M.Sc., Ph.D. degrees from the University of Athens, Greece.

Dr. Tomkos has received the prestigious title of "Distinguished Lecturer" of IEEE Communications Society for the topic of transparent optical networking. Together with his colleagues and students he has authored about 320 peer-reviewed archival articles (over 170 IEEE-sponsored), including about 100 Journal/Magazine/Book publications (about 60 IEEE-sponsored). Dr. Tomkos has served as the Chair of the International "Optical Networking" Technical Committee of IEEE Communications Society and the Chairman of the IFIP working group on "Photonic Networking". He is currently the Chairman of the OSA Technical Group on Optical Communications. He has been General Chair, Technical Program Chair, Subcommittee Chair, Symposium Chair or/and member of the steering/organizing committees for the major conferences (e.g., OFC, ECOC, IEEE GlobeCom, IEEE ICC, ONDM, etc.) in the area of telecommunications/networking (about 100 conferences/symposia/workshops). In addition he is a member of the Editorial Boards of the IEEE/OSA JOURNAL OF LIGHTWAVE TECHNOLOGY, the IEEE/OSA JOURNAL OF OPTICAL COMMUNICATIONS AND NETWORKING, the IET Journal on Optoelectronics, and the International Journal on Telecommunications Management. He is a Fellow of the IET. 